\title{
Double-bundle anterior cruciate ligament reconstruction is superior to single-bundle reconstruction in terms of revision frequency: a study of 22,460 patients from the Swedish National Knee Ligament Register
}

\author{
Eleonor Svantesson ${ }^{2} \cdot$ David Sundemo $^{2} \cdot$ Eric Hamrin Senorski $^{2} \cdot$ Eduard Alentorn-Geli $^{3} \cdot$ Volker Musahl $^{4}$. \\ Freddie H. Fu ${ }^{4}$ Neel Desai ${ }^{1,2}$ - Anders Stålman ${ }^{5}$ Kristian Samuelsson ${ }^{1,2}$
}

Received: 23 August 2016 / Accepted: 15 November 2016 / Published online: 23 November 2016

(c) The Author(s) 2016. This article is published with open access at Springerlink.com

\begin{abstract}
Purpose Studies comparing single- and double-bundle anterior cruciate ligament (ACL) reconstructions often include a combined analysis of anatomic and non-anatomic techniques. The purpose of this study was to compare the revision rates between single- and double-bundle ACL reconstructions in the Swedish National Knee Ligament Register with regard to surgical variables as determined by the anatomic ACL reconstruction scoring checklist (AARSC).

Methods Patients from the Swedish National Knee Ligament Register who underwent either single- or double-bundle ACL reconstruction with hamstring tendon autograft during the period 2007-2014 were included. The follow-up period started with primary ACL reconstruction, and the outcome measure was set as revision surgery. An online questionnaire based on the items of the AARSC was used
\end{abstract}

Kristian Samuelsson

kristian@samuelsson.cc

1 Department of Orthopaedics, Sahlgrenska University Hospital, 43180 Mölndal, Sweden

2 Department of Orthopaedics, Institute of Clinical Sciences, The Sahlgrenska Academy, University of Gothenburg, Gothenburg, Sweden

3 Department of Orthopaedic Surgery, Mayo Clinic, Rochester, MN, USA

4 Department of Orthopaedic Surgery, University of Pittsburgh, Pittsburgh, PA, USA

5 Department of Molecular Medicine and Surgery, Stockholm Sports Trauma Research Center, Karolinska Institutet, Stockholm, Sweden to determine the surgical technique implemented in the single-bundle procedures. These were organized into subgroups based on surgical variables, and the revision rates were compared with the double-bundle ACL reconstruction. Hazard ratios (HR) with $95 \%$ confidence interval (CI) was calculated and adjusted for confounders by Cox regression.

Results A total of 22,460 patients were included in the study, of which 21,846 were single-bundle and 614 were double-bundle ACL reconstruction. Double-bundle ACL reconstruction had a revision frequency of $2.0 \%(n=12)$ and single-bundle 3.2\% $(n=689)$. Single-bundle reconstruction had an increased risk of revision surgery compared with double-bundle [adjusted HR 1.98 (95\% CI $1.12-3.51), p=0.019]$. The subgroup analysis showed a significantly increased risk of revision surgery in patients undergoing single-bundle with anatomic technique using transportal drilling [adjusted HR 2.51 (95\% CI 1.39-4.54), $p=0.002]$ compared with double-bundle ACL reconstruction. Utilizing a more complete anatomic technique according to the AARSC lowered the hazard rate considerably when transportal drilling was performed but still resulted in significantly increased risk of revision surgery compared with double-bundle ACL reconstruction [adjusted HR 1.87 (95\% CI 1.04-3.38), $p=0.037]$.

Conclusions Double-bundle ACL reconstruction is associated with a lower risk of revision surgery than singlebundle ACL reconstruction. Single-bundle procedures performed using transportal femoral drilling technique had significantly higher risk of revision surgery compared with double-bundle. However, a reference reconstruction with transportal drilling defined as a more complete anatomic reconstruction reduces the risk of revision surgery considerably.

Level of evidence III. 
Keywords Anterior cruciate ligament $\cdot$ ACL .

Reconstruction $\cdot$ Revision $\cdot$ Single-bundle $\cdot$ Doublebundle $\cdot$ Revision $\cdot$ Surgery $\cdot$ Anatomic $\cdot$ Checklist

\section{Introduction}

Recent years' knowledge about knee anatomy and kinematics has increased the interest in performing anatomic anterior cruciate ligament (ACL) reconstruction. The ACL consists of at least two distinct functional bundles: the anteromedial bundle and the posterolateral bundle [7]. An anatomic double-bundle ACL reconstruction is therefore close to the native anatomy. Currently though, the single-bundle ACL reconstruction is the most utilized method [22], even though double-bundle reconstruction increases the rotational stability [3, 22, 24, 28, 29]. Several meta-analyses, systematic reviews and a Cochrane review have confirmed superior knee-stability provided by the double-bundle reconstruction, and a Cochrane review showed a trend towards lower re-rupture frequency in favour of the double-bundle reconstruction [3, 13, 17, 22]. However, only two randomized controlled trials report that a double-bundle reconstruction reduces the risk of graft failure [20,21].

Results from two recent register studies showed that the revision rates among single-bundle reconstructions differ depending on the surgical techniques implemented [4, 18]. The studies concluded that single-bundle reconstructions performed using transportal (TP) drilling had an increased risk of revision surgery; however, the results also indicated that there was a learning curve and that a reference reconstruction using TP drilling significantly reduced the revision risk. The anatomic reconstruction demands the use of the TP or outside-in femoral drilling technique which provides visualization of the native footprints and drilling of the femoral and tibial tunnels independent of each other $[10,19]$. The development of anatomic reconstruction has led to questioning of the so long established transtibial (TT) technique since it has been shown to result in non-anatomic positioning of the ACL [14]. The impact of the surgical techniques utilized in a single-bundle reconstruction differs in terms of risk of revision and should thus be considered when comparisons with double-bundle reconstructions are made. A valuable tool when grading and evaluating anatomic ACL reconstructions is the Anatomic Anterior Cruciate Ligament Reconstruction Scoring Checklist (AARSC) [23]. The checklist includes 17 items of importance when performing an anatomic reconstruction where implementation of more items from the AARSC results in a more anatomic reconstruction.

The purpose of this study was to implement the AARSC to a register-based cohort in order to organize single-bundle reconstructions into homogenous groups based on the items fulfilled in the AARSC so that subsequently comparison of the revision rates between single- and double-bundle reconstructions could be made. To date, this is the first national population-based register study to compare single- versus double-bundle reconstruction when also implementing the AARSC. It was hypothesized that the double-bundle reconstruction would be associated with a lower revision frequency than single-bundle.

\section{Materials and methods}

The Swedish National Knee Ligament Register (SNKLR) was used to extract patient data eligible for the study. Data from patients who underwent either single- or doublebundle ACL reconstruction during the period January 1, 2007-December 31, 2014 were included. Only patients aged 13 years or older with primary reconstruction using hamstring tendon autograft were considered eligible. Contralateral knee injury occurring during the followup period was not considered as an exclusion criterion. Full inclusion and exclusion criteria are summarized in Table 1. The date of primary ACL reconstruction marked the start of each patient's follow-up period, which ended on 31 December 2014 or on the date of revision ACL surgery. Primary end point was set as ACL revision surgery. Patients having a possibly shorter follow-up than the earliest documented event (revision ACL surgery) in the specific cohort were censored from analysis, apart from that no minimum follow-up time was pre-specified. Data from individuals undergoing ACL revision surgery were included up until the date of their revision procedure; thus, the postoperative data from these patients was not included in analysis.

Table 1 Inclusion and exclusion criteria

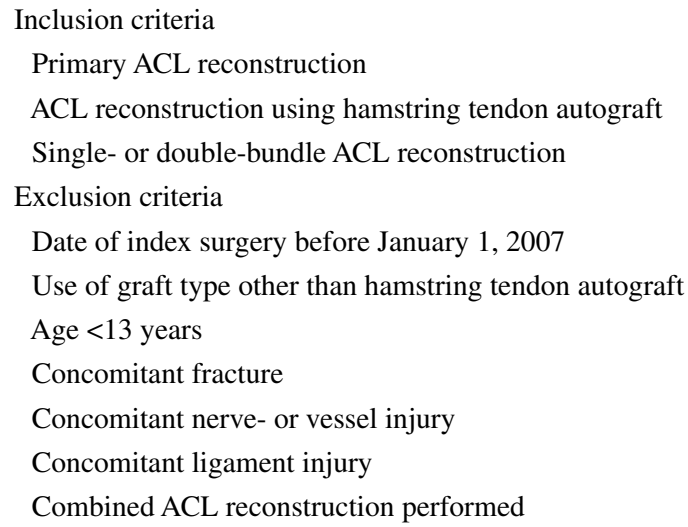




\section{The Swedish National Knee Ligament Register}

The SNKLR is a nationwide database established in January 2005, and $92.9 \%$ of all eligible units for ACL reconstruction in Sweden are linked to the register. Over $90 \%$ of the ACL reconstructions annually performed in Sweden are therefore registered $[6,15]$. The register is sectioned in one data-registration for surgeons and one for patients. Information about activity at the time of injury, date of injury and surgery, fixation method and graft choice is reported. Concomitant injuries, and previous surgery to the knee or the contralateral knee are also registered along with information of all interventions made to the injured knee. Revision surgery or reoperation is separately registered, and the event is correlated with the primary ACL reconstruction. The patient reported outcome is evaluated through two questionnaires, the EQ-5D (European Quality of Life-5 Dimensions) and the KOOS (Knee injury and Osteoarthritis Outcome Score). The patients' response rate preoperatively and at 1,2 and 5 years range from 38 to $72 \%$ [15].

\section{Questionnaire}

Through an online-based questionnaire, detailed information about surgical technique(s) performed by ACL surgeons in Sweden could be collected and matched to a specific ACL reconstruction, a method first described by Desai et al. [4]. The questionnaire was based on the items in the AARSC [23]. All the 175 surgeons registered in the SNKLR as of 31 December 2014 had the possibility to complete the questionnaire online between Jan 2015 and 30 April 2015. The response rate to the questionnaire was $61.7 \%$ [4]. The design of the questionnaire made it possible to identify which surgical technique(s) each surgeon performed during a specific period of time, thereby matching each patient's ACL reconstruction to a specific technique.

\section{Organization of the ACL reconstructions into subgroups}

The single-bundle reconstructions were sorted into groups defined by which surgical techniques had been implemented. In the present study, the same method and predefined groups were used as it was first described by Desai [4]. By combining eight different items from the AARSC, five groups were generated that were characterized by a specific combination of fulfilled items from the AARSC. The combinations of items were selected in order to produce homogenous groups with regard to surgical technique and size. Thus, for a specific ACL reconstruction to be placed in one of the groups the surgeon who performed the reconstruction must have answered to meet the requirements of the group. Each group has at least a few

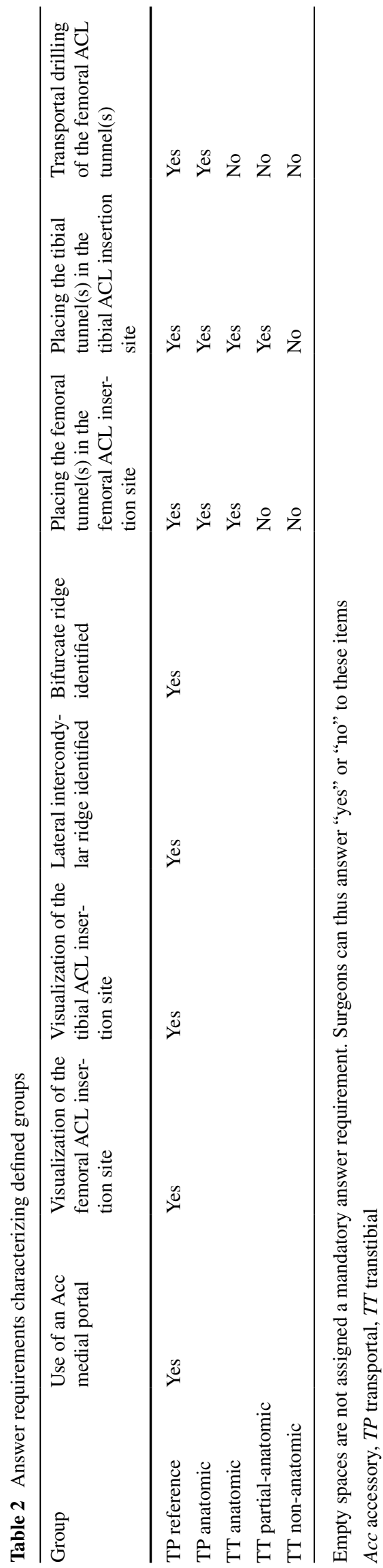


mandatory "yes" or "no" answers regarding the technique used, while some items are considered non-mandatory in some of the groups (Table 2). All single-bundle procedures meeting the inclusion criteria of the study could not be matched with a subgroup since not all surgeons responded to the survey and their surgical technique remains unknown. Furthermore, if a surgeon for any reason could not specify which techniques he or she implemented during a period of time, the reconstructions performed by that surgeon during that interval was not included in a subgroup. The limited amount of double-bundle reconstructions registered during the period of interest made it difficult to further subgroup these reconstructions. The double-bundle group was analysed as one unit, regardless of which surgical techniques had been implemented in the reconstructions included.

This cohort study was conducted according to the WMA Declaration of Helsinki. Participation in the Swedish National Knee Ligament Register is voluntary for patients and surgeons. No written consent is necessary for national databases in Sweden. Investigators had access only to unidentifiable patient data. The study was approved by the Regional Ethics Committee in Gothenburg, Sweden (reference number: 760-14).

\section{Statistical analysis}

Tables and diagrams were generated using Microsoft Excel for Windows (Version 14.0.7, Microsoft Corp, Redmond, Washington, USA). A statistician assigned to the SNKLR performed all statistical analyses. Statistical analysis was performed in IBM SPSS statistics (version 23.0, IBM Corp, Armonk, New York, USA). The data were summarized using counts and percentages for descriptive data and means \pm SDs and median and range for patient-reported outcome data. The end point of revision surgery was analysed as time-to-event outcomes using Cox proportional hazards regression. Kaplan-Meier curves and log minus $\log$ plots were used to visually test the assumption of proportionality. All survival estimates and hazard ratios (HRs) were reported with $95 \%$ confidence intervals (CI). Statistical significance was defined as a $95 \%$ CI for hazard ratios not including 1.0, and alpha was set to 0.05 . Additionally, a multivariate analysis adjusted for possible confounding factors (age and patient sex) was performed using a Cox proportional hazards regression expressed as HR and 95\% CI.

\section{Results}

A total of 22,460 patients were included in the study [12,777 (56.9\%) males and $9683(41.3 \%)$ females]. Patient characteristics are presented in Table 3. Mean 2-year postoperative KOOS score for single- and double-bundle,
Table 3 Baseline patient characteristics

Single-bundle Double-bundle

\begin{tabular}{lcc}
\hline Gender $[N(\%)]$ & & \\
Male & $12,401(56.8)$ & $376(61.2)$ \\
Female & $9445(43.2)$ & $238(38.8)$ \\
Age at index ACL reconstruction $[N(\%)]$ & \\
13-15 years & $1403(6.4)$ & $23(3.7)$ \\
$16-20$ years & $6120(28.0)$ & $165(26.9)$ \\
$21-25$ years & $4435(20.3)$ & $113(18.4)$ \\
$26-30$ years & $3064(14.0)$ & $97(15.8)$ \\
$31-35$ years & $2134(9.8)$ & $71(11.6)$ \\
$36-40$ years & $1910(8.7)$ & $73(11.7)$ \\
$41-45$ years & $1518(6.9)$ & $43(7.0)$ \\
$46-50$ years & $849(3.9)$ & $16(2.6)$ \\
$>51$ years & $413(1.9)$ & $14(2.3)$ \\
Year of surgery $[N(\%)]$ & & \\
2007 & $2183(10.0)$ & $78(12.7)$ \\
2008 & $2380(10.9)$ & $191(31.1)$ \\
2009 & $2586(11.8)$ & $136(22.1)$ \\
2010 & $2884(13.2)$ & $70(11.4)$ \\
2011 & $2896(13.3)$ & $40(6.5)$ \\
2012 & $3072(14.1)$ & $39(6.4)$ \\
2013 & $2.963(13.6)$ & $35(5.7)$ \\
2014 & $2882(13.2)$ & $25(4.1)$ \\
\hline
\end{tabular}

$A C L$ anterior cruciate ligament

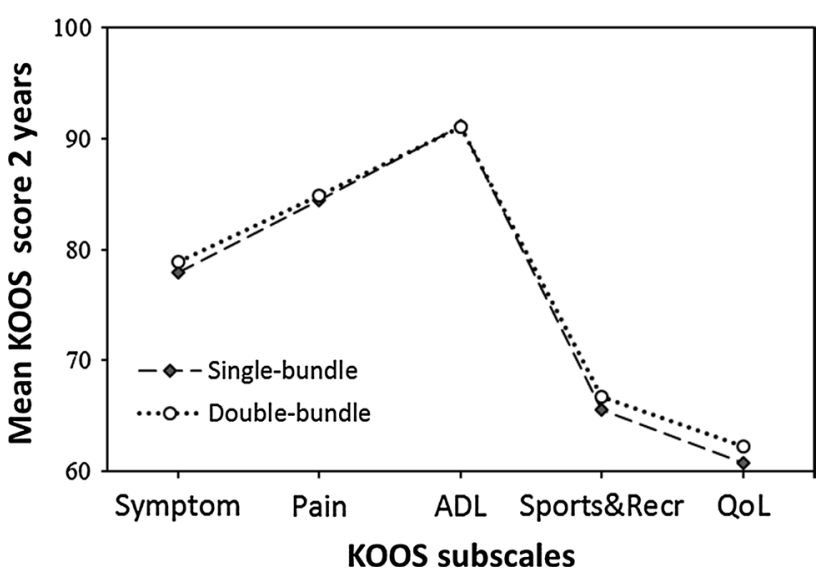

Fig. 1 Mean KOOS scores 2 years after surgery for patients undergoing single-bundle and double-bundle ACL reconstruction, respectively. Please note the scale of the $y$-axis as the scale starts at a KOOS score value of 60. KOOS Knee injury and Osteoarthritis Outcome Score, $A D L$ activities of daily living, Recr recreation, $Q o L$ quality of life

respectively, is found in Fig. 1. The distribution of the date of index ACL surgery for all single-bundle and doublebundle procedures expressed in per cent is presented in Fig. 2. In total $701(3.1 \%)$ revision surgeries were reported between 2007 and 2014 (Table 4). 
Fig. 2 Distribution of date of index ACL surgery for all single-bundle and double-bundle procedures

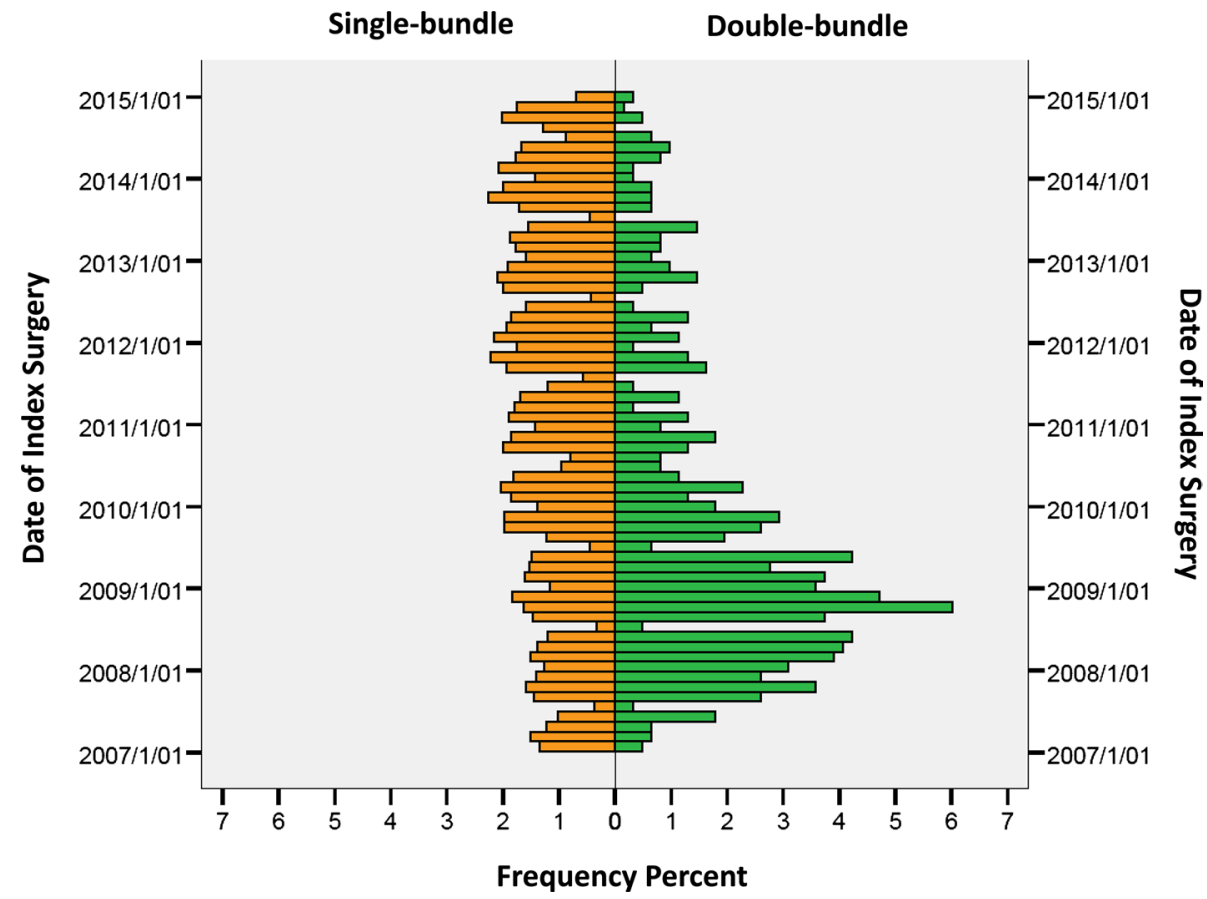

Table 4 Numbers of revision and risk of revision surgery for double-bundle, single-bundle and single-bundle subgroups

\begin{tabular}{|c|c|c|c|c|c|c|c|}
\hline \multicolumn{2}{|l|}{ Groups } & \multicolumn{3}{|c|}{ Crude model } & \multicolumn{3}{|c|}{ Adjusted model ${ }^{\mathrm{a}}$} \\
\hline & No. of events & HR & $95 \% \mathrm{CI}$ & $p$ value & HR & $95 \% \mathrm{CI}$ & $p$ value \\
\hline Double-bundle (reference) $n=614$ & 12 & 1 & & & 1 & & \\
\hline Single-bundle $(n=21,846)$ & 689 & 2.12 & $1.20-3.76$ & 0.010 & 1.98 & $1.12-3.51$ & 0.019 \\
\hline TP reference $(n=5609)$ & 146 & 2.08 & $1.15-3.75$ & 0.015 & 1.87 & $1.04-3.38$ & 0.037 \\
\hline TT non-anatomic $(n=931)$ & 28 & 1.42 & $0.72-2.79$ & $n . s$ & 1.23 & $0.63-2.43$ & $n . s$ \\
\hline TT anatomic $(n=1717)$ & 61 & 1.95 & $1.05-3.63$ & 0.034 & 1.80 & $0.97-3.35$ & $n . s$ \\
\hline TT partial-anatomic $(n=1013)$ & 31 & 1.47 & $0.75-2.86$ & $n . s$ & 1.40 & $0.72-2.73$ & $n . s$ \\
\hline TP anatomic $(n=3449)$ & 133 & 2.66 & $1.47-4.81$ & 0.001 & 2.51 & $1.39-4.54$ & 0.002 \\
\hline Non-classified single-bundle $(n=9127)$ & 209 & & & & & & \\
\hline
\end{tabular}

Numbers of revision and risk of revision surgery; single-bundle subgroups are presented in italics

$H R$ hazard ratio, $C I$ confidence interval, $T P$ transportal, $T T$ transtibial

${ }^{a}$ Cox regression analysis adjusted for patient gender and age at index ACL reconstruction

There was an increased risk of revision surgery for patients who underwent index ACL surgery with single-bundle compared with double-bundle [HR 1.98 (95\% CI 1.12-3.51), $p=0.019]$ adjusted for gender and age at index surgery (Table 4; Fig. 3). In the subgroup analysis, the double-bundle had the highest cumulative survival rates of all groups followed by TT non-anatomic, while the lowest was found in TP anatomic group (Fig. 4). The risk of revision surgery was significantly increased in patients undergoing single-bundle with TP anatomic technique [adjusted HR 2.51 (95\% CI 1.39-4.54), $p=0.002]$, and TP reference surgical technique [adjusted HR 1.87 (95\% CI 1.04-3.38), $p=0.037$ ], compared to double-bundle (Table 4). No significant difference in terms of risk of revision was seen in the other single-bundle subgroups when compared with double-bundle.

\section{Discussion}

The most important finding of present study was that double-bundle reconstruction was associated with a significant lower risk of revision surgery compared with single-bundle reconstruction, confirming the hypothesis of the study. Furthermore, the study showed a distinction in the risk of revision surgery among single-bundle procedures depending on the surgical techniques implemented. 


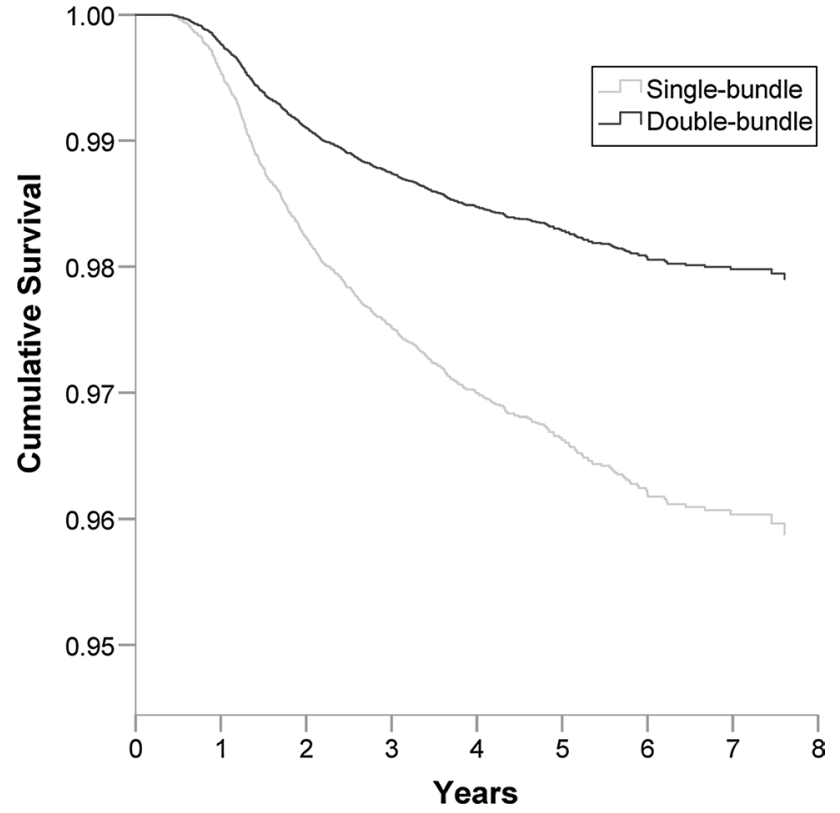

Fig. 3 Cumulative survival function based on Cox proportional hazards regression of single-bundle, double-bundle and revision ACL surgery

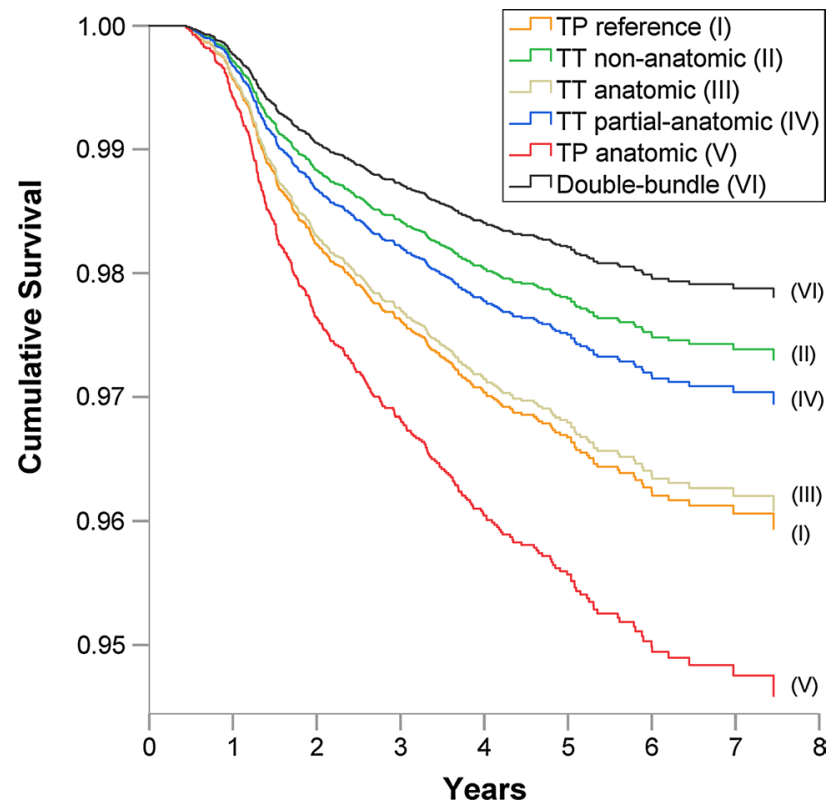

Fig. 4 Cumulative survival function based on Cox proportional hazards regression of single-bundle subgroups, double-bundle and revision ACL surgery. The survival of each single-bundle subgroup is presented by a separate curve

Previous studies of single- versus double-bundle reconstruction often include a combined analysis of anatomic and non-anatomic techniques, and surgical data of the two techniques are grossly underreported in clinical studies $[5,25$,
26]. This study is unique in the sense that it compared the revision rates of single- and double-bundle reconstruction in a large population-based cohort when also taking several surgical variables into consideration. The TP reference group comprised the most anatomic single-bundle reconstructions since all eight items selected from the AARCS were fulfilled. The TP anatomic group was characterized by implementation of only three mandatory techniques. In concordance with previous findings by Desai [4], this study showed that a TP reference technique, defined in terms of a more complete anatomic reconstruction, reduces the risk of revision surgery considerably when the TP drilling technique is utilized. Based on the closely overlapping confidence intervals of the TP reference and the TT anatomic subgroups, the two techniques may be considered equal in terms of risk of revision compared with double-bundle, which is also reported in previous study by Desai [4].

An anatomically placed graft is exposed to higher graft forces $[1,11,16]$ which might predispose graft failure and explain the higher revision frequency among anatomic reconstructions. However, the double-bundle reconstruction more closely resembles the native anatomy where two separately tensioned bundles result in a more naturally distributed graft load during knee range of motion compared with an anatomic single-bundle reconstruction. Along with the greater proportion of femoral and tibial insertion sites covered by the double-bundle, these factors could have positive influence on incorporation, vascularization and maturation of the grafts and explain the lower revision frequency of the double-bundle. By non-anatomic reconstruction the graft load decrease, although several studies have shown that this is done at the expense of rotational stability and function $[11,12,27]$. Following this reasoning, it is worth considering that a double-bundle may have a potential to both distribute graft load properly and provide rotational stability. The ability to better control rotational forces may also directly prevent traumatic re-rupture by counteracting excessive rotational movements of the knee joint.

With today's knowledge about the superiority of the double-bundle reconstruction in terms of restoration of knee joint laxity and the ambition to individualize the procedure for each patient [10], it is likely that patients selected for double-bundle reconstruction have high activity-level demands, great rotational instability and a large size of the knee joint. Assuming this, it is remarkable that only 12 revision surgeries were registered in the group. Furthermore, the double-bundle reconstructions included in this study were performed during a time when the technique was still new to many of the surgeons. The yearly decrease in numbers of double-bundle procedures performed in Sweden may reflect that the procedure is technically challenging, time-consuming and associated with a steep learning curve [17]. It must be emphasized that a 
revision surgery of a double-bundle reconstruction is more complicated than that of a single-bundle reconstruction $[8$, 9]. This fact enhances the possibility that some revision surgeries are not performed in the double-bundle group even though it might be indicated which may result in misleadingly low revision rates in the group. Björnsson et al. [2] have previously investigated the difference in revision rates between single- and double-bundle reconstructions in the SNKLR. Patients undergoing primary ACL reconstruction between 2005 and 2011 were included. In contrast to the present study, no significant differences were found in revision rates between the groups. However, present study includes a much larger patient cohort and a longer followup period.

There are some limitations to this study. First, the limited numbers of double-bundle procedures performed produce large differences in volume of data between the single- and double-bundle cohorts. Consequently, the double-bundle group could not be divided into subgroups with regard to the surgical technique. To do so would have been desirable in order to fully adopt the method of comparing similar surgical techniques with each other. The subgrouping in this study is based on the response-frequency to the questionnaire by the surgeons. There is a risk that reconstructions which may have influenced the analysis of a subgroup were not included in it since their surgical technique was unknown due to non-responding surgeons. The risk of recall bias must be noted as the reliability of the survey depends on correctly reported information about which surgical technique(s) the surgeon implemented during a specific period of time. Also, the primary outcome of revision surgery might withhold the true occurrence of graft failure, and the SNKLR does not provide information about for example patient characteristic, postoperative rehabilitation programme or postoperative activity level which all may influence the outcome of an ACL reconstruction.

Regarding the clinical setting, this study indicates that double-bundle reconstruction is a satisfactorily option and should be considered in the same extent as single-bundle when individualizing treatment. Furthermore, surgeons performing TP drilling should always intend to do so in a thorough way with regard to anatomic technique.

\section{Conclusions}

Double-bundle ACL reconstruction is associated with a lower risk of revision surgery than single-bundle ACL reconstruction. Single-bundle procedures performed using transportal femoral drilling technique had significantly higher risk of revision surgery compared with double-bundle. However, a reference reconstruction with transportal drilling defined as a more complete anatomic reconstruction reduces the risk of revision surgery considerably. The lower revision frequency of the double-bundle reconstruction found in this study indicates the need for further development, utilization and evaluation of this technique.

\section{Compliance with ethical standards}

Conflict of interest The authors declare that they have no conflict of interest.

Funding This research was done under employment at the Sahlgrenska University Hospital and this study received no financial support or grant.

Ethical approval This cohort study was conducted according to the WMA Declaration of Helsinki.

Informed consent No written consent is necessary for national databases in Sweden.

Open Access This article is distributed under the terms of the Creative Commons Attribution 4.0 International License (http://creativecommons.org/licenses/by/4.0/), which permits unrestricted use, distribution, and reproduction in any medium, provided you give appropriate credit to the original author(s) and the source, provide a link to the Creative Commons license, and indicate if changes were made.

\section{References}

1. Araujo PH, Asai S, Pinto M, Protta T, Middleton K, Linde-Rosen M, Irrgang J, Smolinski P, Fu FH (2015) ACL graft position affects in situ graft force following acl reconstruction. J Bone Joint Surg Am 97(21):1767-1773

2. Bjornsson H, Andernord D, Desai N, Norrby O, Forssblad M, Petzold M, Karlsson J, Samuelsson K (2015) No difference in revision rates between single- and double-bundle anterior cruciate ligament reconstruction: a comparative study of 16,791 patients from the Swedish national knee ligament register. Arthroscopy 31(4):659-664

3. Bjornsson H, Desai N, Musahl V, Alentorn-Geli E, Bhandari M, Fu F, Samuelsson K (2015) Is double-bundle anterior cruciate ligament reconstruction superior to single-bundle? a comprehensive systematic review. Knee Surg Sports Traumatol Arthrosc 23(3):696-739

4. Desai N (2016) Anatomic anterior cruciate ligament reconstruction-aspects of surgical technique. Doctoral thesis, Sahlgrenska Academy, University of Gothenburg. http://hdl.handle. net/2077/41829, ISBN: 978-91-628-9625-6

5. Desai N, Alentorn-Geli E, van Eck CF, Musahl V, Fu FH, Karlsson J, Samuelsson K (2016) A systematic review of single- versus double-bundle ACL reconstruction using the anatomic anterior cruciate ligament reconstruction scoring checklist. Knee Surg Sports Traumatol Arthrosc 24(3):862-872

6. Emilsson L, Lindahl B, Koster M, Lambe M, Ludvigsson JF (2015) Review of 103 Swedish healthcare quality registries. J Intern Med 277(1):94-136

7. Girgis FG, Marshall JL, Monajem A (1975) The cruciate ligaments of the knee joint. Anatomical, functional and experimental analysis. Clin Orthop Relat Res 106:216-231 
8. Hofbauer M, Muller B, Murawski CD, Baraga M, van Eck CF, Fu FH (2013) Strategies for revision surgery after primary double-bundle anterior cruciate ligament (ACL) reconstruction. Knee Surg Sports Traumatol Arthrosc 21(9):2072-2080

9. Hofbauer M, Murawski CD, Muller B, van Eck CF, Fu FH (2014) Revision surgery after primary double-bundle ACL reconstruction: aAOS exhibit selection. J Bone Joint Surg Am 96(4):e30

10. Karlsson J, Irrgang JJ, van Eck CF, Samuelsson K, Mejia HA, Fu FH (2011) Anatomic single- and double-bundle anterior cruciate ligament reconstruction, part 2: clinical application of surgical technique. Am J Sports Med 39(9):2016-2026

11. Kato Y, Ingham SJ, Kramer S, Smolinski P, Saito A, Fu FH (2010) Effect of tunnel position for anatomic single-bundle ACL reconstruction on knee biomechanics in a porcine model. Knee Surg Sports Traumatol Arthrosc 18(1):2-10

12. Kondo E, Merican AM, Yasuda K, Amis AA (2011) Biomechanical comparison of anatomic double-bundle, anatomic single-bundle, and nonanatomic single-bundle anterior cruciate ligament reconstructions. Am J Sports Med 39(2):279-288

13. Kongtharvonskul J, Attia J, Thamakaison S, Kijkunasathian C, Woratanarat P, Thakkinstian A (2013) Clinical outcomes of double- vs single-bundle anterior cruciate ligament reconstruction: a systematic review of randomized control trials. Scand J Med Sci Sports 23(1):1-14

14. Kopf S, Forsythe B, Wong AK, Tashman S, Anderst W, Irrgang JJ, Fu FH (2010) Nonanatomic tunnel position in traditional transtibial single-bundle anterior cruciate ligament reconstruction evaluated by three-dimensional computed tomography. J Bone Joint Surg Am 92(6):1427-1431

15. Kvist J, Kartus J, Karlsson J, Forssblad M (2014) Results from the Swedish national anterior cruciate ligament register. Arthroscopy 30(7):803-810

16. Lubowitz JH (2014) Anatomic ACL reconstruction produces greater graft length change during knee range-of-motion than transtibial technique. Knee Surg Sports Traumatol Arthrosc 22(5):1190-1195

17. Mascarenhas R, Cvetanovich GL, Sayegh ET, Verma NN, Cole BJ, Bush-Joseph C, Bach BR Jr (2015) Does double-bundle anterior cruciate ligament reconstruction improve postoperative knee stability compared with single-bundle techniques? A systematic review of overlapping meta-analyses. Arthroscopy 31(6):1185-1196

18. Rahr-Wagner L, Thillemann TM, Pedersen AB, Lind MC (2013) Increased risk of revision after anteromedial compared with transtibial drilling of the femoral tunnel during primary anterior cruciate ligament reconstruction: results from the Danish knee ligament reconstruction register. Arthroscopy 29(1):98-105
19. Song EK, Oh LS, Gill TJ, Li G, Gadikota HR, Seon JK (2009) Prospective comparative study of anterior cruciate ligament reconstruction using the double-bundle and single-bundle techniques. Am J Sports Med 37(9):1705-1711

20. Suomalainen P, Jarvela T, Paakkala A, Kannus P, Jarvinen M (2012) Double-bundle versus single-bundle anterior cruciate ligament reconstruction: a prospective randomized study with 5-year results. Am J Sports Med 40(7):1511-1518

21. Suomalainen P, Moisala AS, Paakkala A, Kannus P, Jarvela T (2011) Double-bundle versus single-bundle anterior cruciate ligament reconstruction: randomized clinical and magnetic resonance imaging study with 2-year follow-up. Am J Sports Med 39(8):1615-1622

22. Tiamklang T, Sumanont S, Foocharoen T, Laopaiboon M (2012) Double-bundle versus single-bundle reconstruction for anterior cruciate ligament rupture in adults. Cochrane Database Syst Rev 11:Cd008413

23. van Eck CF, Gravare-Silbernagel K, Samuelsson K, Musahl V, van Dijk CN, Karlsson J, Irrgang JJ, Fu FH (2013) Evidence to support the interpretation and use of the anatomic anterior cruciate ligament reconstruction checklist. J Bone Joint Surg Am 95(20):e153

24. van Eck CF, Kopf S, Irrgang JJ, Blankevoort L, Bhandari M, Fu FH, Poolman RW (2012) Single-bundle versus double-bundle reconstruction for anterior cruciate ligament rupture: a metaanalysis-does anatomy matter? Arthroscopy 28(3):405-424

25. van Eck CF, Samuelsson K, Vyas SM, van Dijk CN, Karlsson J, Fu FH (2011) Systematic review on cadaveric studies of anatomic anterior cruciate ligament reconstruction. Knee Surg Sports Traumatol Arthrosc 19(Suppl 1):101-108

26. van Eck CF, Schreiber VM, Mejia HA, Samuelsson K, van Dijk CN, Karlsson J, Fu FH (2010) "Anatomic" anterior cruciate ligament reconstruction: a systematic review of surgical techniques and reporting of surgical data. Arthroscopy 26(9 Suppl):S2-12

27. Woo SL, Kanamori A, Zeminski J, Yagi M, Papageorgiou C, Fu $\mathrm{FH}$ (2002) The effectiveness of reconstruction of the anterior cruciate ligament with hamstrings and patellar tendon. A cadaveric study comparing anterior tibial and rotational loads. J Bone Joint Surg Am 84(6):907-914

28. Yagi M, Wong EK, Kanamori A, Debski RE, Fu FH, Woo SL (2002) Biomechanical analysis of an anatomic anterior cruciate ligament reconstruction. Am J Sports Med 30(5):660-666

29. Zelle BA, Vidal AF, Brucker PU, Fu FH (2007) Double-bundle reconstruction of the anterior cruciate ligament: anatomic and biomechanical rationale. J Am Acad Orthop Surg 15(2):87-96 